\title{
ANTITUMOR ACTIVITY OF THE COMBINATION OF BENDAMUSTINE WITH VORINOSTAT IN DIFFUSE LARGE B-CELL LYMPHOMA CELLS
}

Running title: Bendamustine plus vorinostat in lymphoma

Concepción Fernández-Rodríguez ${ }^{1,2}$, Antonio Salar ${ }^{2,3}$, Alfons Navarro ${ }^{4}$, Eva Gimeno ${ }^{2,3}$, Silvia Pairet $^{2,5}$, Laura Camacho ${ }^{2}$, Mariana Ferraro ${ }^{3}$, Sergi Serrano ${ }^{5}$, Carles Besses ${ }^{2,3}$, Beatriz Bellosillo ${ }^{2,5,6}$, Blanca Sanchez-Gonzalez ${ }^{2,3}$.

${ }^{1}$ Universitat Autònoma de Barcelona, Barcelona. ${ }^{2}$ IMIM (Hospital del Mar Medical Research Institute), Barcelona. ${ }^{3}$ Servei d'Hematologia, Hospital del Mar, Barcelona. ${ }^{4}$ Human Anatomy Unit, School of Medicine, Universitat de Barcelona, Barcelona. ${ }^{5}$ Servei de Patologia, Hospital del Mar, Barcelona. ${ }^{6}$ Universitat Pompeu Fabra, Barcelona, Spain.

\section{Corresponding author:}

Blanca Sánchez-González, MD, PhD.

Department of Hematology. Hospital del Mar, Passeig Marítim 25-29, 08003, Barcelona, Spain. Phone and Fax: +34 932483343

E-mail address: 97894@parcdesalutmar.cat

Keywords: bendamustine, vorinostat, diffuse large B-cell lymphoma, TP53, histone deacetylase inhibitor.

- Manuscript word-count: 3396

- Abstract word-count: 102.

- Number of tables and figures: 1 table and 4 figures.

- Number of references: 54. 


\section{ABSTRACT}

Current standard-of-care therapy for diffuse large B cell lymphoma (DLBCL) results in up to $40 \%$ of patients who either relapse or develop refractory disease. In this setting, further therapeutic improvements are needed. Here, we analyzed the in vitro effects of the combination of bendamustine with the histone deacetylase inhibitor vorinostat in DLBCL cells. This combination enhanced histone acetylation and double strand DNA breaks resulting in an additive to synergistic cytotoxic effect in both ABC- and GCB-type DLBCL cells, independently of their TP53 mutational status. These results support the rationale for considering bendamustine and vorinostat combination as a novel approach in DLBCL treatment. 


\section{INTRODUCTION}

Diffuse large B-cell lymphoma (DLBCL) is an aggressive form of non-Hodgkin lymphoma (NHL) that comprises different entities with specific genetic alterations and heterogeneous clinical outcome. Gene expression profiling revealed 3 different molecular subtypes based on cell of origin: germinal center B-cell-like DLBCL (GCB-DLBCL), activated B-cell-like DLBCL (ABCDLBCL), and mediastinal or unclassified type [1]. Although most patients respond to standard treatment with immunochemotherapy (eg, R-CHOP), 40\% remain refractory or relapse, especially within the $A B C$ subgroup [2]. Our increasing knowledge of tumor biology and the identification of targetable pathways can enable the exploration of more effective treatment strategy approaches in DLBCL.

Bendamustine hydrochloride (BEN) was developed as a molecule with alkylator and antimetabolite structure. Compared to other conventional alkylators, such as cyclophosphamide, BEN has a unique mechanistic profile that includes induction of single and double-strand DNA (dsDNA) breaks, activation of DNA damage stress response and apoptosis, inhibition of mitotic checkpoints, and induction of mitotic catastrophe [3]. Moreover, BEN shows limited cross-resistance to other alkylating agents and has demonstrated clinical activity as single-agent therapy or in combination with other antineoplastic agents in relapsed indolent $\mathrm{NHL}$, chronic lymphocytic leukemia (CLL) and multiple myeloma (MM), including patients refractory to alkylating or purine analog agents $[4,5]$. At present time, there is limited data available on the efficacy of BEN in aggressive lymphomas, including DLBCL [6-9].

Vorinostat (VOR) is a histone deacetylase inhibitor (HDACi). HDACis regulate histone and nonhistone protein acetylation, playing a critical role in the modulation of gene expression. Although their specific mechanism of action is still unclear, HDACis exert several antitumor effects, including induction of apoptosis and differentiation, cell cycle arrest, regulation of tumor immunology, tumor suppressor gene transcription, and angiogenesis inhibition [10]. VOR has been approved for the treatment of cutaneous T-cell lymphoma [11] and is currently under study in other types of lymphoma [12-15]. Single-agent vorinostat has shown modest activity in relapsed/refractory indolent NHL (overall response rate -ORR- 29\%), although higher response rates were achieved in patients with follicular histology (ORR 47\%). In aggressive B-cell 
lymphomas, a phase II trial has shown very modest activity for single-agent vorinostat in patients with relapsed DLCBL with only $6 \%$ of cases responding [15]. Preclinical studies have found that HDACis exert their broadest activity in combination with other agents, especially when these combinations are based on the rationale of their mechanistic interaction [16-18]. Thereby, clinical trials using vorinostat plus rituximab or other novel targeted agents have been carried out in some lymphoma types $[19,20]$.

The present study was designed to explore in vitro the interaction of BEN and VOR in DLBCL. We hypothesized that, besides its wide antitumor effects, VOR would promote an open chromatin conformation that might allow extensive BEN accessibility, potentially enhancing the direct DNA damage. To assess this approach, we used two GCB-type and two ABC-type DLBCL cell lines; one of each type harbored known pathogenic TP53 mutations, which affect $20 \%$ of DLBCL patients and are an independent prognostic factor in DLBCL [21].

\section{MATERIALS AND METHODS}

\section{Cell Lines and Primary Cells}

The DLBCL cell line OCI-Ly19 (GCB, TP53 ${ }^{\text {wt }}$ ) was purchased from the DSMZ (Braunschweig, Germany). The SU-DHL-6 (GCB, TP53 ${ }^{\text {mut }}$ ), RC-K8 (ABC, TP53 ${ }^{\text {wt }}$ ) and U-2932 (ABC, TP53 ${ }^{\text {mut }}$ ) were kindly provided by Dr D. Dominguez (Columbia University, NY, USA). Primary cells from a patient with GCB- and from another with ABC-DLBCL (according to Hans' algorithm [22]) were collected by heparinized-RPMI perfusion of fresh neoplastic lymph node biopsies and were stored cryopreserved in liquid nitrogen until use. Both patients gave their signed informed consent. Biological samples were obtained from Parc de Salut MAR Biobank (MARBiobanc) Barcelona. Cell lines and primary cells were grown in RPMI 1640 medium containing $10 \%$ heat-inactivated fetal calf serum, $2 \mathrm{mM} \mathrm{L-glutamine}$ and $50 \mu \mathrm{g} / \mathrm{ml}$ penicillinstreptomycin (GIBCO, Carlsbad, CA, USA) at $37^{\circ} \mathrm{C}$ in a humidified atmosphere containing $5 \%$ carbon dioxide. Main characteristics of all cell lines and primary cells used are summarized in table I.

\section{Chemicals}


VOR (suberoylanilide hydroxamic acid, SAHA) was purchased from Selleck Chemicals (Houston, TX, USA) and BEN was provided by Levact (Mundipharma Pharmaceuticals, Cambridge, UK). VOR was dissolved in $100 \%$ dimetil sulfoxide (DMSO) to a stock concentration of $50 \mathrm{mM}$ and BEN was dissolved in physiological saline at $5 \mathrm{mg} / \mathrm{mL}$.

\section{Analysis of Cell Viability and Apoptosis by Flow Cytometry after Drug Exposure}

Cell lines were incubated $\left(0.5-1 \times 10^{6} / \mathrm{mL}\right)$ with different concentrations of BEN $(25-100 \mu \mathrm{M})$ and/or VOR (0.3-5 $\mu \mathrm{M})$. Cell viability and apoptosis were evaluated by annexin-V staining; the $50 \%$ inhibitory concentration $\left(\mathrm{IC}_{50}\right)$ was defined as the concentration of drug required to reduce cell viability by $50 \%$. Briefly, after 24 and $48 \mathrm{~h}$, cells were washed with annexin binding buffer (100 mM HEPES, $280 \mathrm{mM} \mathrm{NaCl}$ and $5 \mathrm{mM} \mathrm{CaCl}_{2}, \mathrm{pH}$ 7.4), resuspended in $400 \mu \mathrm{L}$ of fresh buffer and labeled for 15 minutes in the dark with annexin-V-FITC (eBioscience, San Diego, CA, USA) at a 1:200 final dilution. The DAPI nucleic acid stain (Invitrogen, Carlsbad, CA, USA) was added (1:4000) at the time of the analysis. Each condition was assessed in duplicates and data were confirmed by at least three independent experiments.

Primary cells were analyzed at $24 \mathrm{~h}$. For cell population selection an anti-CD3 (1:200) and antiCD19 (1:40) antibodies (Becton Dickinson, Franklin Lakes, NJ, USA) were used.

For each analysis, at least 10,000 events were recorded with a BD LSR II flow cytometer (Becton Dickinson). Double-negative population was considered viable cells, while both annexin-V-positive/DAPI-negative and annexin-V-positive/DAPI-positive populations were considered apoptotic committed cells.

\section{Western Blot Analysis}

Proteins were extracted in RIPA buffer (Sigma, St. Louis, MO, USA) and quantified using Bradford assay (Bio-Rad, Hercules, CA, USA). Thirty micrograms of proteins were separated on 10-15\% SDS-PAGE gel and transferred to Immobilion-P PVDF membranes (Millipore, Hayward, CA) and incubated with primary antibodies for anti-acetyl-histone H3 (06-599; 1:20000, Millipore), anti-phospho-H2A.X (07-164, 1:500, Millipore), anti-p53 (NCL-L-p53-DO; 1:1000, Novocastra, Newcastle, UK), anti-poli (ADP-ribose) polymerase (PARP, 11835238 001; 1:2000, Roche, Switzerland) and a-tubulin (CP06; 1:200, Millipore) (used as internal control). 
Appropiate HRP-conjugated secondary antibody (P0260 or P0448; 1:2000; DAKO, Glostrup, Denmark) was used to reveal. Signals were developed with enhanced chemiluminiscence (Pierce, Rockford, IL).

\section{Quantitative Real-Time PCR Analysis Of p21 ${ }^{\mathrm{WAF} 1 / \mathrm{CIP} 1}$ Gene Expression}

After $48 \mathrm{~h}$ of treatment, total cellular RNA from $0.5 \times 10^{6}$ cells was extracted using Trizol (Invitrogen).High Capacity cDNA Reverse Transcription Kit (Applied Biosystems, Foster City, CA, USA), was used for reverse transcription (RT) reactions following the manufacturers' protocols. Primers and probes were purchased from Applied Biosystems (Hs00355782_m1) and analyzed using a 7900 HT Real-Time PCR System (Applied Biosystems). GUS, $\beta$-actine and beta-2-microglobuline were used as internal controls. Relative quantification was calculated using $2^{-\Delta \Delta C t}$.

\section{Cell Proliferation Assay}

Cells were harvested into 96 -well plates $\left(0.1 \times 10^{6}\right.$ per well) and treated with different doses of $\operatorname{BEN}(5,10,25,50,100 \mu \mathrm{M})$ and $\operatorname{VOR}(0.5,1,2,2.5,5 \mu \mathrm{M})$ alone and in all possible combinations. The CellTiter proliferation assay (Promega, Madison, WI) was used according the manufacturer's instructions. The absorbance was measured at $490 \mathrm{~nm}$ with an Infinite 200 PRO microplate reader (Tecan, Durham, NC).

\section{Statistical Analyses}

Means were compared between two groups using a 2-sided Student t-test, using GraphPad Prism 6 (GraphPad Software, Inc., La Jolla, CA, USA). The proliferation and apoptosis data were shown as mean \pm SEM of three independent replicates. To study the interaction between the two drugs we used Bliss independence model. Bliss independence is the index for calculating the expected dose-response relationship for drug combination therapy as compared to mono-therapy, focusing on treatment effect enhancement. Given the two drugs BEN and VOR, both inhibiting tumor growth: BEN at dose $b$ inhibits $Y_{b} \%$ of tumor growth and VOR at dose $v$ inhibits $Y_{v} \%$ of tumor growth. If BEN and VOR work independently, the combined percentage inhibition $Y_{b v, P}$ can be predicted using the complete additivity of probability theory as 
$Y_{b v, P}=Y_{b}+Y_{v}-Y_{b} Y_{v}$. The interaction of each combination of the two drugs can be described by calculating the difference between the predicted $\%$ of growth inhibition, $Y_{b v, P}$, and the observed $\%$ of growth inhibition, $Y_{b v, O}\left(\Delta Y=Y_{b v, O}-Y_{v b, P}\right)$. Therefore, Bliss synergy and antagonism were concluded when $\Delta Y$ and its $95 \%$ confidence interval $(\mathrm{Cl})$ were $>0$ and $<0$, respectively, and in case where the $95 \% \mathrm{Cl}$ of $\Delta Y$ would include 0 , the conclusion was Bliss independence or additive interaction [23].

\section{RESULTS}

\section{BEN and VOR Individually Exert Cytotoxic Effect in DLBCL Cell Lines}

OCI-Ly19 (GCB, TP53 $3^{\text {wt }}$ ), SU-DHL-6 (GCB, TP53 ${ }^{\text {mut }}$ ), RC-K8 (ABC, TP53 $3^{\text {wt }}$ ) and U-2932 (ABC, TP53 ${ }^{\text {mut }}$ ) cells were exposed during 48 hours to increasing doses of BEN or VOR. Concentrations of BEN ranging from 25 to $100 \mu \mathrm{M}$ and of VOR ranging from 0.3 to $2.5 \mu \mathrm{M}$ were used. $I C_{50}$ values were calculated from data obtained from the annexin- $V$ assay performed by flow cytometry (Figure 1 and Table I). For all cell lines, both BEN and VOR alone caused a decrease in cell viability in a dose-dependent manner. Each cell line showed a different sensitivity to BEN although no association was found with DLBCL subtypes, whereas GCBDLBCL cell lines showed a higher cytotoxic response to VOR than ABC-DLBCL subtype $(\mathrm{P}<0.001)$.

\section{BEN and VOR In Combination Treatment}

For the combination assays, the $\mathrm{IC}_{50}$ of $\mathrm{BEN}$ and a fixed dose of VOR of $2.5 \mu \mathrm{M}$ were used. $\mathrm{IC}_{50}$ concentrations and achieved toxicity for each cell type are listed in Table I. Both drugs were added simultaneously. As shown in Figure 2A, the addition of VOR resulted in a significant increase of cytotoxicity compared to that obtained with BEN alone. The Bliss model for drug combination study indicated that the cytotoxic effects were slightly synergistic for the SU-DHL-6 and the RC-K8 cell lines and additive for the other two cell lines at the doses tested (Table I).

\section{Effect of the Sequence of Treatment}

To explore a potential enhanced cytotoxic effect of pretreating cells with VOR, we assessed different sequences of treatment. However, the addition of BEN 24 or $72 \mathrm{~h}$ after VOR did not 
increase the cytotoxic effect compared to the concomitant exposure to both drugs (data not shown).

\section{Effects in Primary Cells}

The effects of BEN and VOR, alone and in combination, were studied in the subpopulation of lymph node B cells (CD3-, CD19+) from 2 DLBCL (1 GCB and 1 ABC) patients. At 24h, both BEN and VOR showed a dose-dependent effect on cell viability. The drug combination induced a higher cytotoxic response comparing with the individual effects of BEN and VOR alone drugs but no significant differences in cytotoxicity were observed according to $A B C / G C B$ subtype (Figure 2B)

\section{dsDNA Breaks and Apoptosis Induction}

To study the ability of BEN and VOR to induce DNA damage, H2A.X phosphorylation was analyzed as an indirect marker of dsDNA breaks. As shown in Figure 3, no signal was detected in untreated cells after 48 hours incubations, whereas both BEN and VOR induced dsDNA breaks. When cells were treated with the combination of both drugs, a 1.6- to 5.5-fold increase in H2A.X phosphorylation was seen compared to BEN exposure alone.

The enzymatic cleavage of PARP protein, a classic marker for caspase 3-induced apoptosis, was evaluated by western blot (Figure 3). After $48 \mathrm{~h}$ of BEN and/or VOR treatment, the $85-\mathrm{kDa}$ corresponding to cleaved PARP was detected. PARP cleavage was slightly higher after BEN treatment than after the combination treatment.

\section{Histone H3 Acetylation}

Since histone $\mathrm{H} 3$ is one of the main substrates of HDACis, $\mathrm{H} 3$ acetylation was assessed by western blot. Hyperacetylation of histone $\mathrm{H} 3$ was observed following VOR treatment (Figure 3). Interestingly, BEN alone induced only weak detectable levels of acetylated $\mathrm{H} 3$ in two cell lines, whereas the combination of BEN and VOR resulted in a 2.7- to 5.6-fold increase of histone $\mathrm{H} 3$ acetylation in three out four cell lines compared to VOR treatment alone.

p53 and p21 Expression 
In the western blot study, after BEN treatment, a 1.3- and a 3.3-fold increase of p53 were observed in the OCI-Ly19 and RC-K8 TP53-wild-type cells, respectively (Figure 4A), whereas VOR decreased p53 levels in all cells. After the combination treatment, only the RC-K8 cells showed a 2.3 fold increase of p53, while the other cells showed slightly decreased p53 expression. We next examined $\mathrm{p} 21^{\mathrm{WAF} 1 / \mathrm{CIP} 1}$ expression by quantitative PCR. BEN treatment triggered a notable increase in p21 ${ }^{\mathrm{WAF} 1 / \mathrm{CIP} 1}$ in both TP53-wild-type cells OCI-Ly19 and RC-K8, matching the p53 results. VOR treatment enhanced p21 $1^{\text {WAF1/CIP1 }}$ expression in the two GCBsubtype cell lines OCI-Ly19 and SU-DHL-6, while it remained invariable in ABC-subtype cells. After the BEN-plus-VOR combination treatment, p21 ${ }^{\mathrm{WAF} 1 / \mathrm{CIP} 1}$ increased in the OCI-Ly19 and SUDHL-6 cells and slightly in the RC-K8 cells.

\section{DISCUSSION}

As a complex multi-hit disease, DLBCL shows a wide range of clinical and molecular features that result in heterogeneous clinical responses. Distinct pathogenic pathways have been described to be altered in these patients, supporting the efforts to explore new drug combination strategies [24]. Phase I and II clinical trials in DLBCL patients containing BEN $[8,9,25,26]$ or VOR $[14,15]$ have been reported, showing diverse responses. Despite of BEN has shown promising results, VOR in monotherapy has shown limited activity in DLBCL patients. However, there are no clinical trials using the combination of both drugs and the mechanisms of action of both drugs alone or in combination have been poorly explored in DLBCL. [3,4,16,27-31].

In our study, we have analyzed the cytotoxic effects of BEN and VOR as single agents and in combination in DLBCL cells and we have studied the mechanisms involved in this process. At clinically achievable concentrations, both BEN and VOR exerted single-agent cell killing activity in a dose- and time-dependent manner against GCB and ABC-DLBCL cell lines as well as in patients' cells in ex vivo cultures. Interestingly, GCB-type cell lines were more sensitive to VOR than ABC-type cells, whereas no difference in sensitivity was observed to BEN treatment. This differential response to VOR is opposed to the results obtained in a prior report using the panHDACi belinostat with some of the same cell lines used in our study, which found that cells' sensitivity did not depend on the DLBCL-subtype [32]. Hyperacetylation of NF-KB in response to HDACis has been proposed to cause an anti-apoptotic effect $[10,16]$, which would explain the 
lower sensitivity to VOR in ABC-DLBCL cell lines, that are known to depend on the constitutive activation of the NF-KB pro-survival factor, in contrast to the GCB-DLBCL type [33].

Novel targeted therapies such as BCR inhibitors are known to affect ABC-DLBCL cell by affecting the NF-kB signaling pathway. For instance, ibrutinib, a BTK-inhibitor, in combination with VOR have shown synergistic effect in mantle cell lymphoma cell lines [19]. Moreover, VOR potentiates the activity of carfilzomib, a proteasome inhibitor, in GC- and ABC-DLBCL cells, including bortezomib-resistant cells [16]. However, the activity of BEN in combination with VOR in DLBCL subtypes is limited and deserves further investigation. Other specific agents such as monoclonal antibodies against CD20 can be used to target B-cell lymphomas. Of interest, a phase 2 study using a combination of rituximab plus VOR has shown promising efficacy in patients with indolent non-Hodgkin lymphoma [20]. This combination might be also useful for patients with DLBCL but clinical trials are still ongoing.

In order to explore the contribution of an HDACi to BEN mode of action, a fixed dose of VOR was added. The addition of a $2.5 \mu \mathrm{M}$ dose of VOR [34] to the BEN treatment of DLBCL cell lines resulted in a significantly higher toxicity comparing with BEN treatment as single-agent (Figure 1). This increased toxic effect was synergistic in two cell lines (SUDHL6 and RC-K8), while it was more likely to be independent or additive in the others, according to the Bliss independence model. Apart from their single-agent antitumor effects, HDACis act remodeling chromatin to a loosen conformation [10], potentially making the DNA more accessible to damaging agents. Here, we show a striking increase of phosphorylated-H2A.X (P-H2A.X) levels when co-treating cells with BEN and VOR, thus demonstrating enhanced DNA damage [35]. Regarding this, prior reports have provided evidence about the importance of the sequence of treatment when combining HDACis with DNA-damaging agents [36-39], but in our study VOR pre-treatment exposure did not enhance BEN cytotoxic effect compared to the addition of both drugs concomitantly, in accordance with a previous study in leukemia cells of our group [40]. On the other hand, in this study we have demonstrated that the ability of VOR to promote acetylation was enhanced when cells were treated together with BEN, even though BEN alone showed a very weak acetylation effect. A recent study reported the synergistic histone acetylation when treating lymphoma cells with bendamustine and the HDACi romidepsin together, leading to an increased toxicity, but the underlying mechanisms behind this effects are 
still unknown [41]. Common alterations affecting genes that play a role in epigenetic regulation have been recently described in DLBCL, such as those affecting the HATs CREBBP and EP300, that are known to be mutated in a $32 \%$ of DLBCLs [42-44]. Mutant forms of these two genes have been shown to cause an impaired acetylation that may contribute to lymphomagenesis by over-expression of the main DLBCL-related oncogene BCL6 and inactivation of p53 $[45,46]$. Inactivating mutations in EP300 and/or CREBBP are known to affect the HAT function of SU-DHL-6 and OCI-Ly19 cells whereas RC-K8 presents a well characterized rearrangement in EP300 $[45,47]$. As we have demonstrated in this study, VOR and more powerfully BEN plus VOR combination, enhanced histone acetylation in these defective cells. Thus, the use of VOR and BEN combined could potentially revert this situation as part of their anti-lymphoma mechanisms.

One fifth of all DLBCL patients harbor somatic mutations in the TP53 gene, which correlate with poor survival to R-CHOP standard treatment, even within GCB and ABC subtypes [21]. The p53 protein is a crucial tumor suppressor that mediates cell-cycle arrest, DNA repair, apoptosis, senescence and autophagy in response to cellular stress [48]. In order to explore the role of the TP53 mutational status in the BEN and VOR combination treatment, two out of the four cell lines used in this study carried deleterious TP53 mutations. In our case, TP53 mutational status did not correlate with differences in viability and apoptosis after BEN and VOR exposure, which implies that both drugs are effective in DLBCL cells independently of the TP53 mutational status. However, p53 protein levels were differentially modulated according to TP53 status and to the cytotoxic treatment, revealing that different mechanisms of apoptosis may be driving the cytotoxic effect in each situation. BEN exposure resulted in an increase of p53 protein levels and p21 upregulation only in the TP53-wild type cell lines, meaning that the cytotoxicity observed in cells with a nonfunctional p53 involved p53-independent mechanisms, such as the previously postulated mitochondrial oxidative stress or mitotic catastrophe $[3,49]$. On the other hand, it is well described that wild type p53 is not necessary for HDACis induced apoptosis [50], since cell cycle arrest through p53-independent induction of p21 is a major mechanism of VOR induced cytotoxicity $[10,34,51]$. In our experiments, VOR decreased the expression p53 in all cases, but only GCB cells had increased p21 expression, further correlating with the higher cytotoxic achieved effects. Intermediate amounts of p53 and cleaved PARP were seen in the 
BEN plus VOR combination treatment, corroborating that HDACis and p53-activating agents may cause p53 antagonistic regulation in wild type-p53 cells, rather than increase the antitumor efficacy [50]. However, increased levels of p21 expression were maintained, and DNA damage levels, histone acetylation and apoptosis were enhanced, meaning that opposed regulation of p53 is overcome by these and potentially other mechanisms. Published data indicate that sensitivity of NHL cells to treatment with HDACis is dependent on the complex regulation of BCL-2 antiapoptotic family members, that includes the accumulation of MCL-1 as well as other inhibitors of apoptosis and the downregulation of BCL-X, among others [31,52,53], The accumulation of antiapoptotic regulators would cause a decrease in the apoptosis levels, whereas the drop of BCL-X after HDACi treatment has been described to activate the apoptosis inducing factor (AIF)-dependent apoptotic pathway, that is independent of caspase activation and PARP cleavage [54]. These two events, which we have also observed in our cells (data not shown) could explain the reduction of p53 and cleaved PARP without compromising cytotoxic effects.

In summary, we have demonstrated that the pan-HDAC inhibitor VOR potentiates the BENinduced cytotoxicity in both GCB and ABC-DLBCL cells, independently on their TP53mutational status. Given the favorable toxicity profiles of both drugs, their distinctive mechanism of action and the favorable perspective of new drug combination approaches, the combination of BEN and VOR may be promising for the management of DLBCL patients with relapsed or refractory disease, even those with TP53 mutations. 


\section{CONFLICT OF INTEREST STATEMENT}

The authors declare no conflict of interest.

\section{ACKNOWLEDGEMENTS}

This work was supported by grants from AECC Cataluña 2009, 2014 SGR 567, Instituto de Salud Carlos III FEDER (PT13/0010/0005) and the "Xarxa de Bancs de tumors" sponsored by Pla Director d'Oncologia de Catalunya (XBTC). Concepción Fernández-Rodríguez received a fellowship from the Ministry of Economy and Competitiveness of Spain (PFIS grant Fl11/00353).

Contributions: CF, AN, AS, CB, and BB contributed to the design; CF, SP and LC performed the experiments; CF, AS and AN analyzed and interpreted the data; BB designed and reviewed molecular assessment; EG and MF selected cases and provided relevant intellectual content; SS performed histopathological review of patients' biopsies; CF, and AN wrote the manuscript; BS contributed to the concept and design, planned the study and critically reviewed the manuscript; all authors participated in discussions, provided scientific input and gave the approval of the final manuscript.

\section{REFERENCES}

[1] Alizadeh A, Eisen M, Davis R, Ma C. Distinct types of diffuse large B-cell lymphoma identified by gene expression profiling. Nature 2000;403:503-11.

[2] Cheson BD. Therapy for diffuse large B-cell lymphoma: getting personal. Lancet 2013;6736:1-2.

[3] Leoni LM, Bailey B, Reifert J, Bendall HH, Zeller RW, Corbeil J, et al. Bendamustine (Treanda) displays a distinct pattern of cytotoxicity and unique mechanistic features compared with other alkylating agents. Clin Cancer Res 2008;14:309-17.

[4] Korycka-Wołowiec A, Robak T. Pharmacokinetic evaluation and therapeutic activity of bendamustine in B-cell lymphoid malignancies. Expert Opin Drug Metab Toxicol 2012;8:1455-68. 
[5] Rummel MJ, Niederle N, Maschmeyer G, Banat GA, von Grünhagen U, Losem C, et al. Bendamustine plus rituximab versus CHOP plus rituximab as first-line treatment for patients with indolent and mantle-cell lymphomas: an open-label, multicentre, randomised, phase 3 non-inferiority trial. Lancet 2013;381:1203-10.

[6] Friedberg JW, Cohen P, Chen L, Robinson KS, Forero-Torres A, La Casce AS, et al. Bendamustine in patients with rituximab-refractory indolent and transformed nonHodgkin's lymphoma: results from a phase II multicenter, single-agent study. J Clin Oncol 2008;26:204-10.

[7] Sanchez-Gonzalez B, Garcia M, Serrano S, Besses C, Salar A. Bendamustinecontaining immunochemotherapy is active in transformed follicular lymphoma with overexpression of p53. Leuk Lymphoma 2009;50:455-6.

[8] Walter E, Schmitt T, Dietrich S, Ho A, Witzens-Harig M. Rituximab and bendamustine in patients with CD20+ diffuse large B-cell lymphoma not eligible for cyclophosphamide, doxorubicin, vincristine and prednisone-like chemotherapy. Leuk Lymphoma 2012;53:2290-2.

[9] Ohmachi K, Niitsu N, Uchida T, Kim SJ, Ando K, Takahashi NN, et al. Multicenter Phase II Study of Bendamustine Plus Rituximab in Patients With Relapsed or Refractory Diffuse Large B-Cell Lymphoma. J Clin Oncol 2013;31:2103-9.

[10] Bolden JE, Peart MJ, Johnstone RW. Anticancer activities of histone deacetylase inhibitors. Nat Rev Drug Discov 2006;5:769-84.

[11] Mann BS, Johnson JR, Cohen MH, Justice R, Pazdur R. FDA approval summary: vorinostat for treatment of advanced primary cutaneous T-cell lymphoma. Oncologist 2007;12:1247-52.

[12] Kirschbaum M, Frankel P, Popplewell L, Zain J, Delioukina M, Pullarkat V, et al. Phase II study of vorinostat for treatment of relapsed or refractory indolent non-Hodgkin's lymphoma and mantle cell lymphoma. J Clin Oncol 2011;29:1198-203. 
[13] Richardson PG, Mitsiades CS, Laubach JP, Hajek R, Spicka I, Dimopoulos M a, et al. Preclinical data and early clinical experience supporting the use of histone deacetylase inhibitors in multiple myeloma. Leuk Res 2013;37:829-37.

[14] O'Connor OA, Heaney ML, Schwartz L, Richardson S, Willim R, MacGregor-Cortelli B, et al. Clinical experience with intravenous and oral formulations of the novel histone deacetylase inhibitor suberoylanilide hydroxamic acid in patients with advanced hematologic malignancies. J Clin Oncol 2006;24:166-73.

[15] Crump M, Coiffier B, Jacobsen ED, Sun L, Ricker JL, Xie H, et al. Phase II trial of oral vorinostat (suberoylanilide hydroxamic acid) in relapsed diffuse large-B-cell lymphoma. Ann Oncol 2008;19:964-9.

[16] Dasmahapatra G, Lembersky D, Kramer L, Fisher RI, Friedberg J, Dent P, et al. The pan-HDAC inhibitor vorinostat potentiates the activity of the proteasome inhibitor carfilzomib in human DLBCL cells in vitro and in vivo. Blood 2010;115:4478-87.

[17] Zuco V, De Cesare M, Cincinelli R, Nannei R, Pisano C, Zaffaroni N, et al. Synergistic antitumor effects of novel HDAC inhibitors and paclitaxel in vitro and in vivo. PLoS One $2011 ; 6: \mathrm{e} 29085$.

[18] Bodo J, Zhao X, Sharma A, Hill BT, Portell CA, Lannutti BJ, et al. The phosphatidylinositol 3-kinases (PI3K) inhibitor GS-1101 synergistically potentiates histone deacetylase inhibitor-induced proliferation inhibition and apoptosis through the inactivation of PI3K and extracellular signal-regulated kinase pathways. $\mathrm{Br} \mathrm{J}$ Haematol 2013;163:72-80.

[19] Hagiwara K, Kunishima S, lida H, Miyata $Y$, Naoe T, Nagai H. The synergistic effect of BCR signaling inhibitors combined with an HDAC inhibitor on cell death in a mantle cell lymphoma cell line. Apoptosis 2015. 
[20] Chen R, Frankel P, Popplewell L, Siddiqi T, Ruel N, Rotter A, et al. A phase II study of vorinostat and rituximab for treatment of newly diagnosed and relapsed/refractory indolent non-Hodgkin lymphoma. Haematologica 2015;100:357-62.

[21] Xu-Monette ZY, Wu L, Visco C, Tai YC, Tzankov A, Liu W, et al. Mutational profile and prognostic significance of TP53 in diffuse large B-cell lymphoma patients treated with RCHOP: report from an International DLBCL Rituximab-CHOP Consortium Program Study. Blood 2012;120:3986-96.

[22] Hans CP, Weisenburger DD, Greiner TC, Gascoyne RD, Delabie J, Ott G, et al. Confirmation of the molecular classification of diffuse large B-cell lymphoma by immunohistochemistry using a tissue microarray. Blood 2004;103:275-82.

[23] BLISS Cl. THE TOXICITY OF POISONS APPLIED JOINTLY1. Ann Appl Biol 1939;26:585-615.

[24] Wilson WH. Treatment strategies for aggressive lymphomas: what works? Hematology Am Soc Hematol Educ Program 2013;2013:584-90.

[25] Weidmann E, Kim S-Z, Rost A, Schuppert H, Seipelt G, Hoelzer D, et al. Bendamustine is effective in relapsed or refractory aggressive non-Hodgkin's lymphoma. Ann Oncol 2002;13:1285-9.

[26] Vacirca JL, Acs PI, Tabbara IA, Rosen PJ, Lee P, Lynam E. Bendamustine combined with rituximab for patients with relapsed or refractory diffuse large B cell lymphoma. Ann Hematol 2013;93:403-9.

[27] Kaneko N, Mitsuoka K, Amino N, Yamanaka K, Kita A, Mori M, et al. Combination of YM155, a Survivin Suppressant, with Bendamustine and Rituximab: A New Combination Therapy to Treat Relapsed/Refractory Diffuse Large B-cell Lymphoma. Clin Cancer Res 2014;20:1814-22.

[28] Beeharry N, Rattner JB, Bellacosa A, Smith MR, Yen TJ. Dose dependent effects on cell cycle checkpoints and DNA repair by bendamustine. PLoS One 2013;7:e40342. 
[29] Sakajiri S, Kumagai T, Kawamata N, Saitoh T, Said JW, Koeffler HP. Histone deacetylase inhibitors profoundly decrease proliferation of human lymphoid cancer cell lines. Exp Hematol 2005;33:53-61.

[30] Cai $Y$, Cui W, Chen W, Wei $P$, Chi $Y$, Zhang $P$, et al. The effects of a histone deacetylase inhibitor on biological behavior of diffuse large B-cell lymphoma cell lines and insights into the underlying mechanisms. Cancer Cell Int 2013;13:1-9.

[31] Thompson RC, Vardinogiannis I, Gilmore TD. The Sensitivity of Diffuse Large B-Cell Lymphoma Cell Lines to Histone Deacetylase Inhibitor-Induced Apoptosis Is Modulated by BCL-2 Family Protein Activity. PLoS One 2013;8:e62822.

[32] Tula-Sanchez AA, Havas AP, Alonge PJ, Klein ME, Doctor SR, Pinkston W, et al. A model of sensitivity and resistance to histone deacetylase inhibitors in diffuse large B cell Iymphoma: Role of cyclin-dependent kinase inhibitors. Cancer Biol Ther 2013;14:949-61.

[33] Davis RE, Brown KD, Siebenlist U, Staudt LM. Constitutive Nuclear Factor kB Activity Is Required for Survival of Activated B Cell-like Diffuse Large B Cell Lymphoma Cells. J Exp Med 2001;194:1861-74.

[34] Xu WS, Parmigiani RB, Marks PA. Histone deacetylase inhibitors: molecular mechanisms of action. Oncogene 2007;26:5541-52.

[35] Mah L-J, El-Osta A, Karagiannis TC. gammaH2AX: a sensitive molecular marker of DNA damage and repair. Leukemia 2010;24:679-86.

[36] Kim MS, Blake M, Baek JH, Kohlhagen G, Pommier Y, Carrier F. Inhibition of Histone Deacetylase Increases Cytotoxicity to Anticancer Drugs Targeting DNA. Cancer Res 2003;63:7291-300.

[37] Blagosklonny M V, Trostel S, Kayastha G, Demidenko ZN, Vassilev LT, Romanova LY, et al. Depletion of mutant p53 and cytotoxicity of histone deacetylase inhibitors. Cancer Res 2005;65:7386-92. 
[38] Marchion DC, Bicaku E, Daud AI, Richon V, Sullivan DM, Munster PN. Sequencespecific potentiation of topoisomerase II inhibitors by the histone deacetylase inhibitor suberoylanilide hydroxamic acid. J Cell Biochem 2004;92:223-37.

[39] Ageberg M, Rydström K, Relander T, Drott K. The histone deacetylase inhibitor valproic acid sensitizes diffuse large B-cell lymphoma cell lines to CHOP-induced cell death. Am J Transl Res 2013;5:170-83.

[40] Sanchez-Gonzalez B, Yang H, Bueso-Ramos C, Hoshino K, Quintas-Cardama A, Richon VM, et al. Antileukemia activity of the combination of an anthracycline with a histone deacetylase inhibitor. Blood 2006;108:1174-82.

[41] Hiraoka N, Kikuchi J, Koyama D, Wada T, Mori S, Nakamura Y, et al. Alkylating agents induce histone H3K18 hyperacetylation and potentiate HDAC inhibitor-mediated global histone acetylation and cytotoxicity in mantle cell lymphoma. Blood Cancer $\mathrm{J}$ 2013;3:e169.

[42] Morin RD, Mendez-Lago M, Mungall AJ, Goya R, Mungall KL, Corbett RD, et al. Frequent mutation of histone-modifying genes in non-Hodgkin lymphoma. Nature 2011;476:298-303.

[43] Jiang Y, Hatzi K, Shaknovich R. Mechanisms of epigenetic deregulation in lymphoid neoplasms. Blood 2013;121:4271-9.

[44] Pasqualucci L, Dalla-Favera R. SnapShot: Diffuse Large B Cell Lymphoma. Cancer Cell 2014;25:132-132.e1.

[45] Pasqualucci L, Dominguez-Sola D, Chiarenza A, Fabbri G, Grunn A, Trifonov V, et al. Inactivating mutations of acetyltransferase genes in B-cell lymphoma. Nature 2011;471:189-95.

[46] Phan RT, Dalla-Favera R. The BCL6 proto-oncogene suppresses p53 expression in germinal-centre B cells. Nature 2004;432:635-9. 
[47] Garbati MR, Alço G, Gilmore TD. Histone acetyltransferase p300 is a coactivator for transcription factor REL and is C-terminally truncated in the human diffuse large B-cell lymphoma cell line RC-K8. Cancer Lett 2010;291:237-45.

[48] Vousden KH, Lane DP. P53 in Health and Disease. Nat Rev Mol Cell Biol 2007;8:27583.

[49] Roué G, López-Guerra M, Milpied P, Pérez-Galán P, Villamor N, Montserrat E, et al. Bendamustine is effective in p53-deficient B-cell neoplasms and requires oxidative stress and caspase-independent signaling. Clin Cancer Res 2008;14:6907-15.

[50] Yan W, Liu S, Xu E, Zhang J, Zhang Y, Chen X. Histone deacetylase inhibitors suppress mutant p53 transcription via histone deacetylase 8. Oncogene 2013;32:599-609.

[51] Richon VM, Sandhoff TW, Rifkind RA, Marks PA. Histone deacetylase inhibitor selectively induces p21WAF1 expression and gene-associated histone acetylation. Proc Natl Acad Sci U S A 2000;97:10014-9.

[52] Inoue S, Riley J, Gant TW, Dyer MJS, Cohen GM. Apoptosis induced by histone deacetylase inhibitors in leukemic cells is mediated by Bim and Noxa. Leukemia 2007;21:1773-82.

[53] Henderson C, Mizzau M, Paroni G, Maestro R, Schneider C, Brancolini C. Role of caspases, Bid, and p53 in the apoptotic response triggered by histone deacetylase inhibitors trichostatin-A (TSA) and suberoylanilide hydroxamic acid (SAHA). J Biol Chem 2003;278:12579-89.

[54] Hajji N, Wallenborg K, Vlachos P, Nyman U, Hermanson O, Joseph B. Combinatorial action of the HDAC inhibitor trichostatin A and etoposide induces caspase-mediated AIFdependent apoptotic cell death in non-small cell lung carcinoma cells. Oncogene 2008;27:3134-44. 
Table I. Characteristics of DLBCL cell lines and primary cells.

\begin{tabular}{|c|c|c|c|c|c|}
\hline DLBCL cells & phenotype & TP53 & $\begin{array}{l}\text { bendamustine } \\
\text { sensitivity 48h } \\
\text { IC } 50(\mu \mathrm{M})\end{array}$ & $\begin{array}{c}\text { vorinostat } \\
\text { cytotoxicity } 48 \mathrm{~h} \\
2.5 \mu \mathrm{M}(\%)\end{array}$ & $\begin{array}{l}\text { Bliss independence }^{\mathrm{a}} \\
\text { bendamustine } \mathrm{IC}_{50} \\
\text { +vorinostat } 2.5 \mu \mathrm{M}\end{array}$ \\
\hline OCl-Ly19 & GCB & $w t^{b}$ & 5 & 53.3 & $-0.020 \pm 0.188$ \\
\hline SU-DHL-6 & GCB & $\begin{array}{c}\text { p.Y234Y/C } \\
\text { p.R306L }\end{array}$ & 75 & 61.7 & $0.107 \pm 0.030$ \\
\hline RC-K8 & $A B C$ & wt & 100 & 32.5 & $0.072 \pm 0.054$ \\
\hline U-2932 & $A B C$ & $\begin{array}{l}\text { p.C176Y } \\
\text { p.L344L/P }\end{array}$ & 50 & 31.1 & $0.041 \pm 0.066$ \\
\hline Tumor sample \#1 & GCB & wt & $25^{\dagger}$ & $41.5^{\mathrm{C}}$ & - \\
\hline Tumor sample \#2 & $A B C$ & wt & $25^{\dagger}$ & $59.6^{c}$ & - \\
\hline
\end{tabular}

${ }^{a}$ Difference between observed and predicted cytotoxic effect according to the Bliss model and confidence interval

${ }^{b}$ Wild type

${ }^{\mathrm{c}} 24 \mathrm{~h}$ of drug exposition 


\section{FIGURE LEGENDS}

Figure 1. Bendamustine and vorinostat exert cytotoxicity in GCB and ABC-DLBCL cells, independently of their TP53 mutational status. U-2932, RC-K8, SU-DHL-6 and OCI-Ly19 cells were treated with increasing concentrations of bendamustine $(5,25,50,100 \mu \mathrm{M})$ or vorinostat $(1,2.5,5 \mu \mathrm{M})$ during $48 \mathrm{~h}$. Cells were analyzed by FACS following staining with annexin-V/DAPI. The percentage of viable cells was normalized considering untreated control cells viability at $48 \mathrm{~h}$ as $100 \%$.

Figure 2. Combined treatment of bendamustine and vorinostat increases their cytotoxic effect as single agents. Apoptotic cells where determined by annexin-V/DAPI staining in three independent experiments. Mean percentages of unviable cells \pm SEM are represented. ${ }^{*} p<0.05$, ${ }^{* *} \mathrm{p}<0.01$, NS: non-significant. (A) OCI-Ly19, SU-DHL-6, RC-K8 and U-2932 cells where treated with bendamustine $\left(\mathrm{IC}_{50}\right)$, vorinostat $(2.5 \mu \mathrm{M})$ or their combination during $48 \mathrm{~h}$. (B) DLBCL primary cells were treated with bendamustine $\left(\mathrm{IC}_{50}\right)$, vorinostat $(1 \mu \mathrm{M})$ or their combination during $24 \mathrm{~h}$.

Figure 3. Bendamustine and vorinostat combination enhances DNA damage and histone H3 acetylation. Cells were treated with VOR at $2.5 \mu \mathrm{M}$ and BEN at $100 \mu \mathrm{M}(5 \mu \mathrm{M}$ of BEN in OCI-Ly19 cells, which had shown a greater sensitivity) during $48 \mathrm{~h}$. Western blot analyses of cleaved PARP, phosphorylated H2A.X and acetylated histone H3 were performed on the cell lysates from the four cell lines. The levels of $\beta$-actin served as loading control.

Figure 4. Bendamustine and vorinostat trigger cytotoxic response in a different manner. After exposition to the indicated doses, p53 (A) and p21 ${ }^{\text {WAF1/CIP1 }}$ (B) expression were assessed by western blot and quantitative PCR, respectively. Three independent experiments were performed. Western blot results shown are representative of three independent experiments. For the quantitative PCR assay, average percentages \pm SEM are represented. ${ }^{*} p<0.05$, ${ }^{* *} p<0.01$. 
FIG1

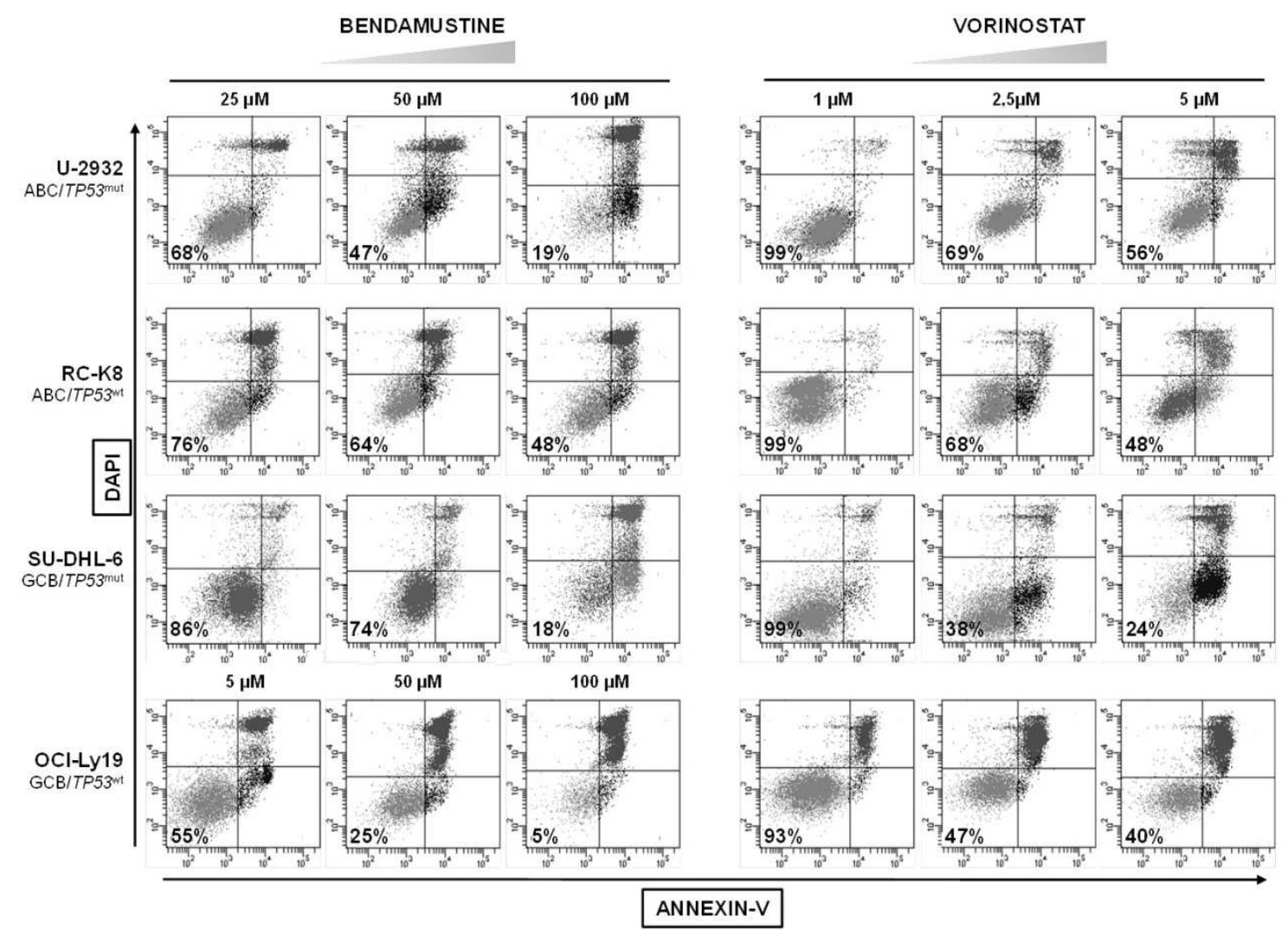


FIG2

A
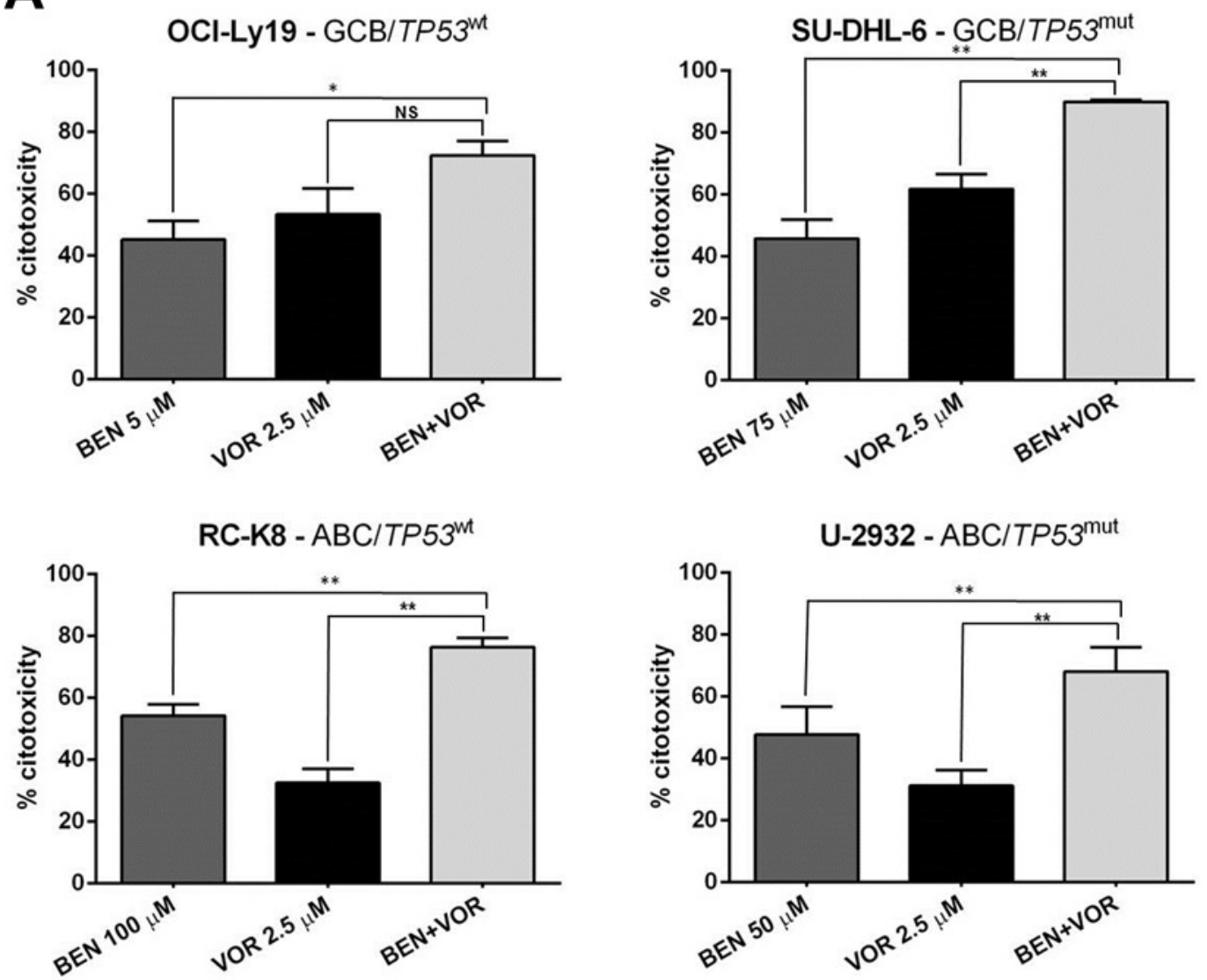

B

PRIMARY SAMPLES
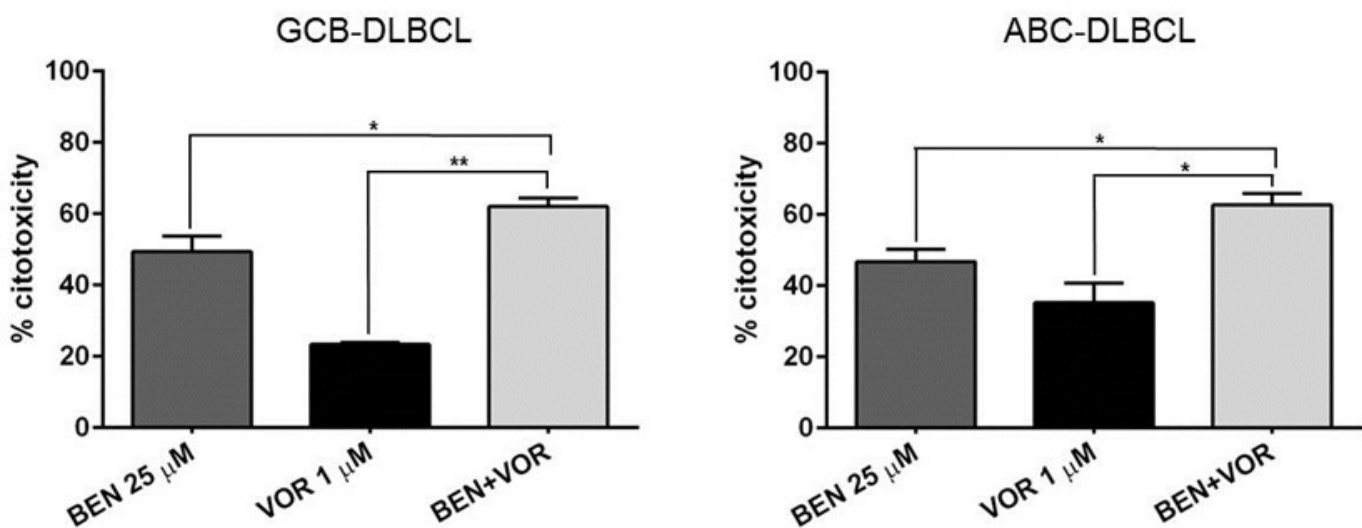
FIG3

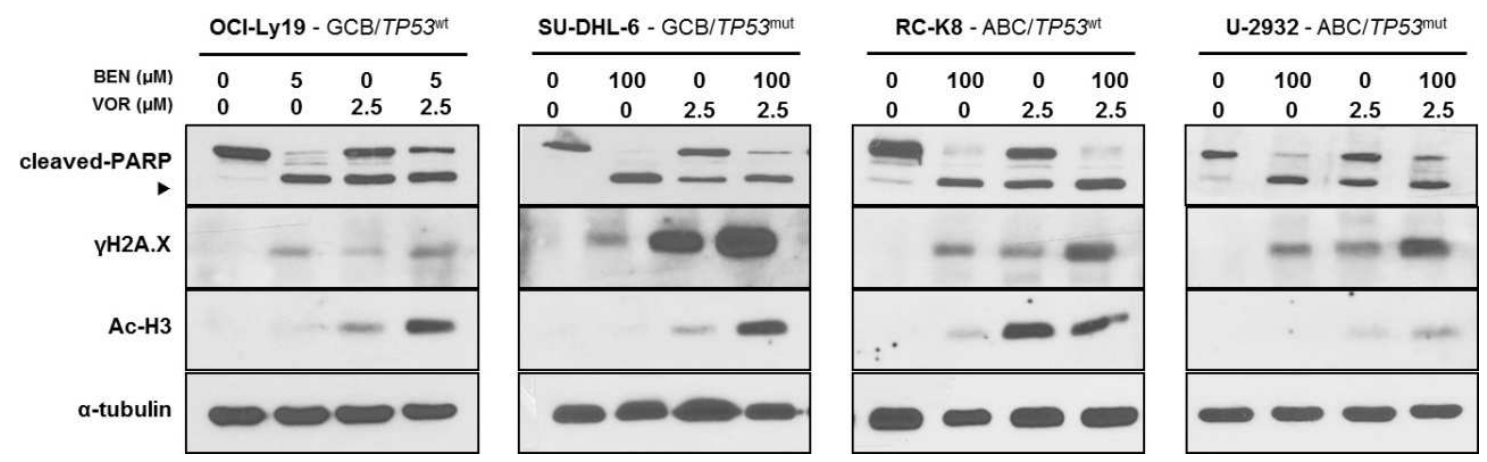

FIG4

A OCI-Ly19 - GCB/TP53wt SU-DHL-6 - GCB/TP53mut RC-K8 - ABC/TP53 ${ }^{\text {wt }}$ U-2932 - ABC/TP53mut
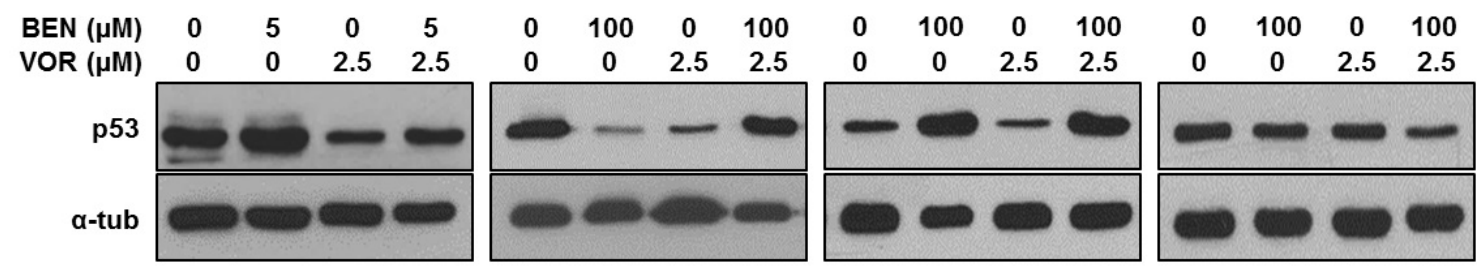

B

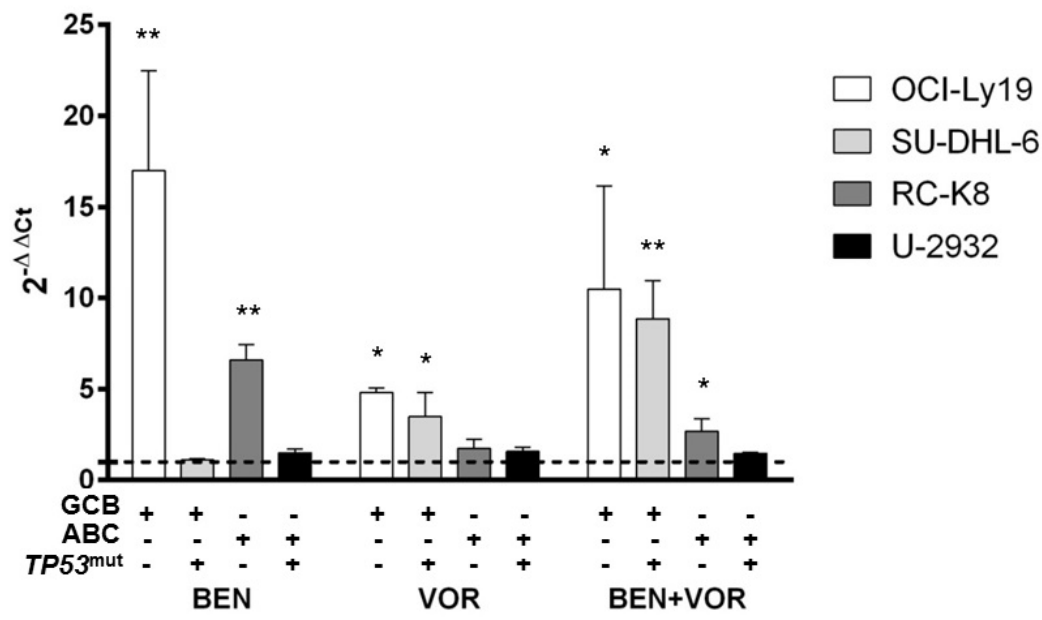

